\title{
Juegos en tabletas digitales como introducción al modelado y la impresión 3D
}

\section{Serious games for digital tablets as an introduction to 3D modeling and printing}

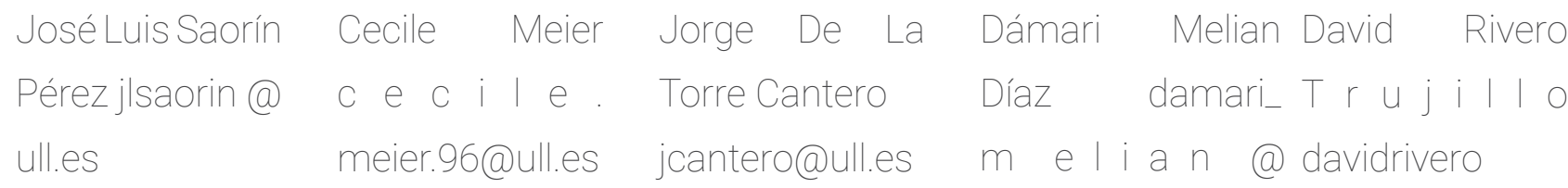

hotmail.com trujil|o@ hotmail.com

\section{Universidad de la Laguna, España}

Fecha de recepción: 28-12-2014

Fecha de revisión: 26-03-2015

\section{Palabras clave:}

Videojuego; Aprendizaje colaborativo;

Aplicaciones; Ipad; Impresión 3D.
Fecha de aceptación: 11-05-2015

Fecha de publicación: 15-06-2015

\section{Resumen}

Keywords:

Apps; Ipad; 3D Printing; 3D Modeling;

Education; Serious Games.

En la actualidad, una de las formas más directas por la que los niños acceden a la tecnología digital es mediante los videojuegos. Este aspecto no suele ser considerado en entornos educativos en la adquisición de Competencias Básicas. Sin embargo, existen informes internacionales que valoran la potencialidad de los videojuegos como recurso educativo. En este sentido, desde el 2006, el Informe Horizon incluye los videojuegos educativos como una tecnología con repercusión en la enseñanza, el aprendizaje y la expresión creativa dentro de los entornos educativos. En este mismo informe, a partir del 2012 las
Currently, one of the most direct ways children access to digital technology is through games. This aspect is usually not considered in educational settings in the acquisition of Basic Skills. However, there are international reports assessing the potential of video games as an educational resource. Since 2006, the Horizon Report includes educational video games as a technology impact on teaching, learning and creative expression in education environments. In the 2012 Report, Digital Tablets are considered as one of the technologies most likely to be in widespread use in education in the short 
Tabletas Digitales son consideradas como una de las tecnologías con mayor posibilidad de ser de uso generalizado en educación a corto plazo. Por otro lado, existen estudios que relacionan mayor interés de alumnos en carreras de ciencia, arte y tecnología al uso prematuro de herramientas de modelado o impresión 3D. En este artículo queremos poner en valor dos aplicaciones, Blokify y Pottery, disponibles para tabletas digitales, que funcionan como juegos y que introducen al alumno en el modelado y la impresión tridimensional digital.

\section{Introducción}

Existe una corriente educativa, (gamification), que trata de potenciar la motivación, la concentración, el esfuerzo y otros valores comunes a todos los juegos, para influir y motivar a los alumnos. Con dos y tres años de edad los niños comienzan a utilizar los dispositivos móviles tipo tabletas y smartphones de sus padres para entretenerse con los juegos. El 52,5\% de los menores de 11 a 14 años de edad juega habitualmente con sus dispositivos móviles y el 35,5 \% lo hace en alguna ocasión, solo un $11 \%$ afirma no hacerlo nunca (Cánovas, García de Pablo, Oliaga San Atilano, \& Aboy Ferrer, 2014). El informe Horizon 2013 (Horizon, 2013) indica que las tabletas digitales y las impresoras 3D son tecnologías que tendrán impacto en la educación en los próximos cinco años. Las tabletas digitales y dispositivos móviles son usados por el $30 \%$ de los niños españoles term. On the other hand, there are studies linking student interest in careers in science, art and technology to the premature use of 3D modeling tools or 3D printing. In this article, we want to value two applications, Blokify and Pottery, available for digital tablets, which work like games that introduce students to the three-dimensional digital modeling and printing.

de 10 años de edad. A los 12 años, casi el $70 \%$ dispone ya de este tipo de tecnología, y a los 14 años el 83 \% (Cánovas, García de Pablo, Oliaga San Atilano, \& Aboy Ferrer, 2014). Uno de los estudios más extensos sobre tabletas "The iPad as a tool for education - a case study" (Heinrich, 2012) demuestra el impacto significativo y muy positivo en la enseñanza y en el aprendizaje de los alumnos. Las tabletas digitales, debido a su portabilidad y su autonomía, permiten convertir cualquier aula en un espacio digital, eliminado la problemática asociada a aulas de ordenadores.

Por otro lado, las impresoras 3D son máquinas controladas por ordenador que permiten generar objetos mediante adición de material utilizando para ello diferentes tecnologías (plástico fundido, resina fotosensible, etc.); a esta tecnología se la conoce también como 
prototipado rápido (Canessa, Fonda, \& Zennaro, 2013). El abaratamiento de esta tecnología en los últimos años permite pensar en su uso para contextos educativos.

En este artículo se van a analizar dos aplicaciones para tabletas digitales: Blokify y Pottery. La primera de ellas, Blokify, que permite modelar en 3D mediante cubos, con un modo de funcionamiento que recuerda a la construcción con lego. La otra aplicación es Pottery, que imita el modelado de barro

\section{Antecedentes}

El modelado y la impresión 3D, hasta hace aproximadamente ocho años, eran tecnologías reservadas para expertos en la materia y requerían un largo y costoso aprendizaje, además se precisaba un equipo técnico avanzado y el precio de los programas era muy elevado y solo accesible para grandes centros, empresas o universidades (Caño, de la Cruz, \& Solano, 2007).

La tecnología de la impresión 3D se empezó a utilizar en los años setenta y desde su aparición, las universidades han tratado de utilizarlas como complemento a su docencia. Desde hace años, el prototipado rápido es un tema habitual en los cursos y en los libros de expresión gráfica dirigidos a estudiantes universitarios. Pero uno de los grandes factores limitantes del uso de esta tecnología en las aulas ha sido el precio. En el año 2000 una máquina de prototipado rápido tenía un precio que oscilaba entre 10.000 y 100.000 dólares, por lo que solo los grandes centros en un torno con posterior decoración de las creaciones. Por lo tanto, los dos juegos que tratamos en este artículo son de modelado tridimensional y debido a que funcionan en tabletas digitales, pueden ser aplicados en contextos educativos para asignaturas de dibujo y artes plásticas. Es interesante señalar que ambas aplicaciones cuentan con la posibilidad de imprimir los diseños en una impresora 3D.

podían permitirse el lujo de tener una.

Sin embargo, en el año 2005 se crea el proyecto RepRap con el objetivo de abaratar los costes asociados a la impresión 3D. Esta iniciativa dio lugar a la popularización de dichas máquinas, ya que los costes asociadas a fabricar una de ellas eran del orden de los 1000 dólares. Uno de los fundadores del proyecto RepRap, Zach Smith, fundó en 2009 la empresa MakerBot, cuyo objetivo era vender kits de impresoras 3D para que todo el mundo pudiera montarse su propia impresora por menos de 1000 dólares. A partir de ese momento, las impresoras de bajo coste se han popularizado y ha surgido una industria alrededor de ellas. La aparición de esta nueva gama de impresoras permite a la mayoría de los centros educativos disponer de una de ellas y utilizarla en su docencia. Por lo tanto, una vez superado la barrera del precio, es necesario disponer de metodologías y recursos docentes que nos permitan sacar 
partido de las impresoras 3D en entornos educativos (Canessa, Fonda, \& Zennaro, 2013).

Respecto al modelado 3D, en el año 2006 se hizo popular el programa Sketchup gracias a su distribución gratuita por la empresa Google. Sketchup es un programa de libre acceso, multiplataforma (PC y Mac) y de descarga gratuita que nos ofrece la posibilidad de introducirnos en el Modelado 3D con pocos conocimientos y en muy poco tiempo. Dispone de una interfaz amigable, con un reducido número de órdenes intuitivas unido a una sencillez de manejo que propicia un rápido aprendizaje. Debido a estas características, este programa se ha utilizado en entornos educativos en materias relacionadas con el dibujo y para la mejora de la visión espacial (de la Torre Cantero J., Saorín, Carbonell, Del Castillo Cossío, \& Contero, 2012).

Otras empresas que desarrollan software de modelado tridimensional están empezando a desarrollar programas con esta nueva manera de entender el modelado 3D. Por ejemplo, Autodesk, que ha desarrollado programas y aplicaciones gratuitas y sencillas de aprender y manejar. Agrupados dentro de una suite llamado 123Dapp. Cada una de ellas sirve para un modelado específico, por un lado más geométrico como 123D Design o más orgánico como 123D Creature. Todas estas cuentan con la posibilidad de imprimir los diseños en una impresora 3D.

Estas aplicaciones tienen una versión desarrollada para tabletas digitales y por ello son ideales para introducirlas en cualquier aula, sin necesidad de recurrir a aulas específicas de ordenadores. Estas tecnologías de pantallas portátiles con las funciones de ordenador llevan tiempo desarrollándose. Los primeros aparatos que podemos llamar tabletas digitales surgieron en el año 93, llamadas Apple Message Pad, más conocido como Newton. El modelo nunca llegó a tener éxito, pero sin embargo el desarrollo del software que se utilizó por la industria de la electrónica sirvió de base para crear un nuevo aparato electrónico denominado Personal Digital Assitant (PDA). En el año 2001 Microsoft presenta diversos prototipos de Tablet's Pc, pero el funcionamiento y la experiencia de uso de estos dispositivos no logró convencer a la mayoría de los usuarios. Sin embargo, esta iniciativa de Microsoft popularizó el terminó Tablet PC y sentó las bases de la corriente actual de tabletas digitales. La empresa Apple lanzó en 2010 la primera tableta digital realmente operativa, el iPad, que aprovechaba la experiencia de la empresa con los dispositivos móviles táctiles que ya tenía en el mercado (el iPhone, el iPod Touch) (Escuelapedia, 2014).

Respecto al uso de videojuegos en educación, ya en 1978 G. Ball publicó el artículo "Telegames Teach More Than You Think" (Ball, 1978) en el que estableció cuatro áreas para la evaluación de los videojuegos como medios didácticos: el desarrollo instructivo de los videojuegos, el desarrollo de habilidades por parte de los videojuegos, el diseño de los videojuegos y su capacidad de adaptabilidad y flexibilidad. Uno de los 
estudios sobre el potencial instructivo de los juegos, realizado por B. Lowery y F. Knirk (1982), más concretamente sobre los videojuegos, destaca la mejora en habilidades espaciales y el beneficio de la simulación tridimensional, aspecto también fundamental de la visualización espacial. En España, en la Universidad de La Laguna, existe una experiencia que utiliza el videojuego Tetris como herramienta educativa para la mejora de la visión espacial (Saorín, Martín Gutiérrez, Martín Dorta, \& Contero, 2009).

En 1984 S. Long y W. Long (Long \& Long, 1984) defienden los principios del aprendizaje motivador que subyacen en los videojuegos: el desafío, la fantasía y la curiosidad. Poco después, S. Silvern (Silvern, 1985-86) publicó un artículo titulado "Classroom Use of Video Games" en el que, de igual modo, defendió todo aquello que los videojuegos pueden ofrecer en términos de experiencias educativas útiles.

En 1998 se publica el libro "Jugando con Videojuegos: educación y entretenimiento" (Gros, 1998), acerca de la experiencia en la utilización de videojuegos en el aula. La publicación de 2004 "Pantallas, juegos y educación: la alfabetización digital en la escuela" (Gros, 2004), es la construcción de bases sólidas para la integración de las TIC (tecnologías de la información y la comunicación) en la educación. En 2011 el Instituto de Tecnologías Educativas, del Departamento de Proyectos Europeos, publica el proyecto Imagine, "Juegos digitales para el aprendizaje" (Instituto de Tecnologías Educativas, 2011). Su objetivo es aumentar la experimentación del aprendizaje basado en juegos y la integración de éste en las enseñanzas generales del sistema educativo.

\section{Descripción de las aplicaciones Blokify y Pottery}

Blokify es un juego, gratuito, disponible para tabletas iPad que permite construir figuras mediante el uso de bloques en forma de cubos. Dispone únicamente de dos funciones, una es colocar bloques y la otra eliminarlos. La interfaz del juego es tipo tablero de ajedrez y cuenta con un entorno adaptado para los niños. Prácticamente no es necesario un aprendizaje, solo se necesitan las instrucciones básicas de cómo rotar el espacio y moverse en el entorno de modelado tridimensional (Figura 1). Además la aplicación cuenta con la posibilidad de imprimir en 3D los modelados tridimensionales.

La otra aplicación es Pottery, un juego que imita el proceso de creación de figuras de arcilla en un torno. Se trabaja a base de presionar con el dedo en la pantalla y moldear mediante revolución la arcilla del torno. También permite decorar las figuras con diferentes colores o pinceles. A medida que avanzamos en el juego, "vendiendo" nuestras creaciones, se consiguen puntos para comprar más colores y nuevas decoraciones 

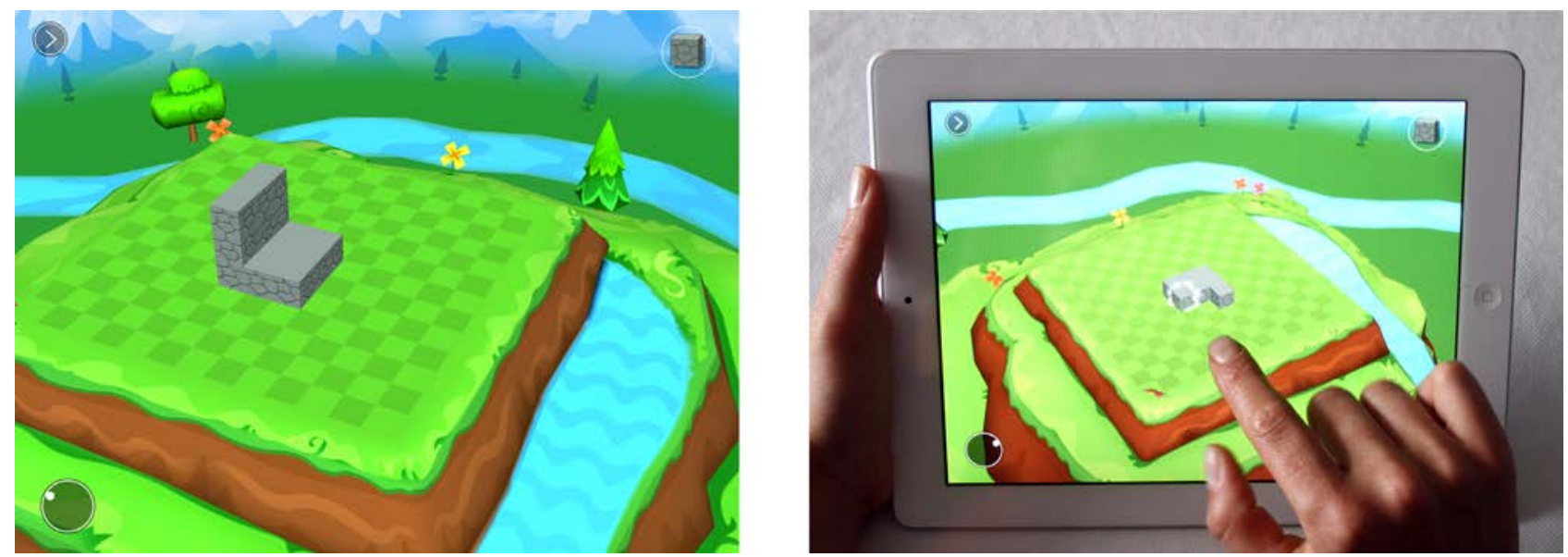

Figura 1: interfaz de la aplicación Blokify.

(Figura 2). Esta aplicación cuenta con la aunque solamente enviándolos por mail a una posibilidad de imprimir en 3D los diseños, empresa de servicios de impresión 3D.
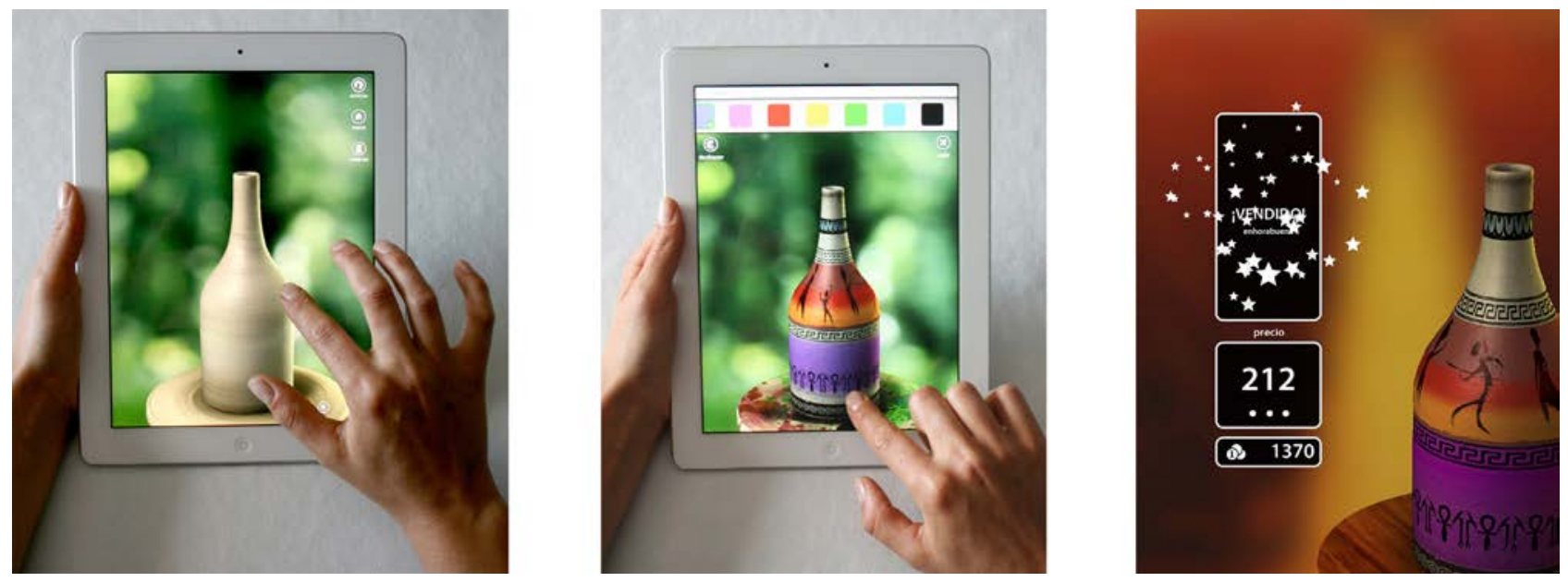

Figura 2: Funcionamiento de la aplicación Pottery.

\section{Descripción de las actividades}

\subsection{Blokify}

Una de las actividades que se pueden normalizadas y los objetos en perspectiva se desarrollar con Blokify es la introducción basan en ejercicios sobre papel que incluye a las vistas normalizadas como planta, dibujos en los que los alumnos deben alzado y perfil. La enseñanza de las vistas identificar las figuras y saber sacar las vistas 
normalizadas. O al revés, sacar el dibujo en la elección de carreras más técnicas (Wai, tres dimensiones a partir de las tres vistas. Lubinski, \& Benbo, 2009). Estas carreras La interpretación de los gráficos es compleja son más conocidas bajo las iniciales STEM y requiere conocimientos específicos y (Science, Technology, Engineering, and habilidades espaciales (Diezmann \& Lowrie, Mathematics), estudios de ciencia, tecnología, 2009), complicados en un principio para los ingeniería y matemáticas. Consideradas en alumnos. 2011, por los organismos estadounidenses
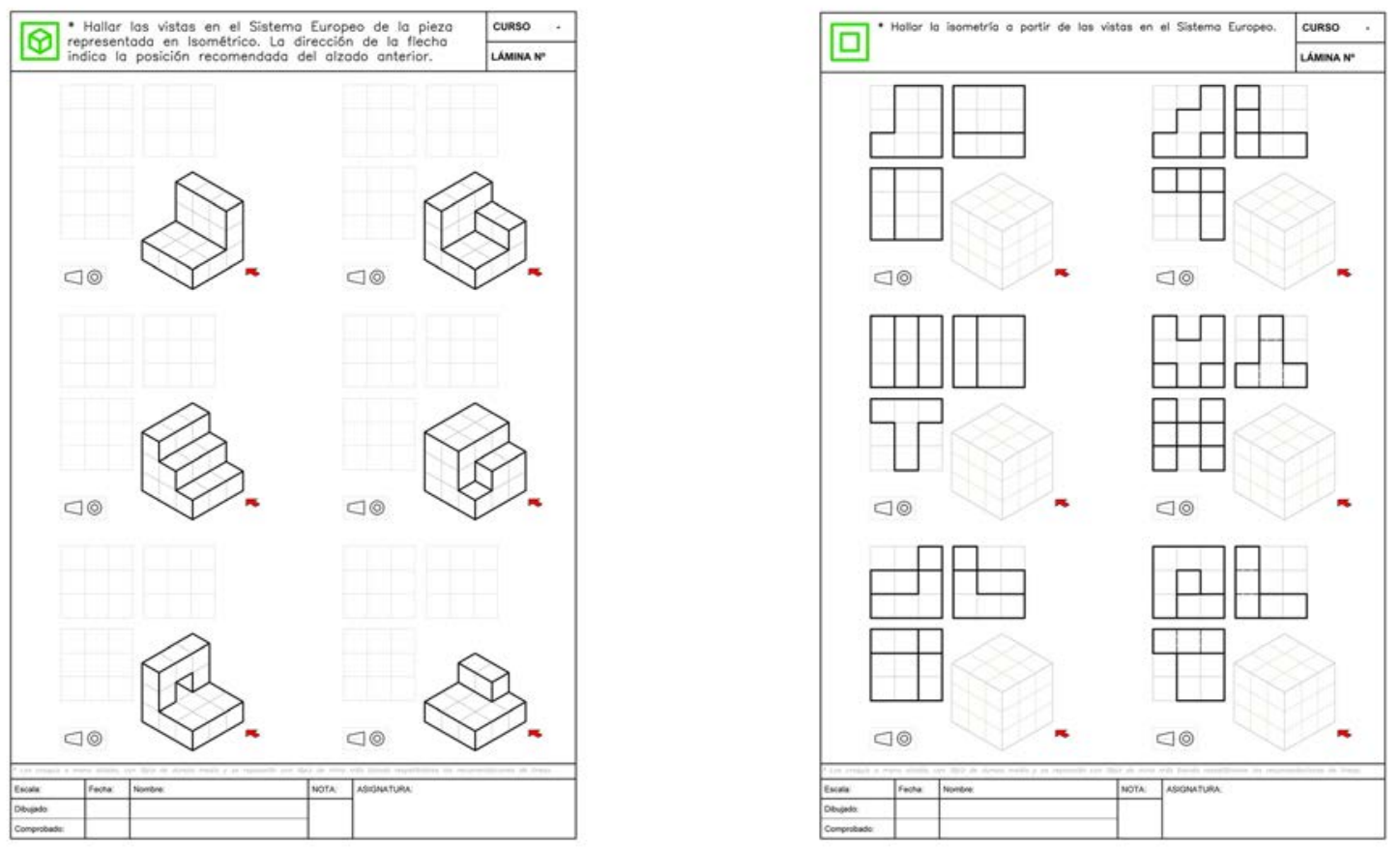

Figura 3: Ejercicios de Perspectivas y vistas normalizadas.

El desarrollo de las habilidades espaciales es un objetivo presente en los currículos a partir de la Educación Secundaria. Incluye las relaciones espaciales y la percepción, visión y rotación espacial (Sjölinder, 1998). En la Educación Primaria estos objetivos están relacionados con el espacio físico que rodea al niño (Holloway, 1982).

Está demostrado que las habilidades espaciales se desarrollan mediante entrenamiento (de la Torre Cantero J. , Saorín, Carbonell, Del Castillo Cossío, \& Contero, 2012). Y se relaciona mejor conocimiento y facilidad de asimilación de las vistas normalizadas con del United States National Research Council y la National Science Foundation, disciplinas fundamentales para las sociedades tecnológicamente avanzadas e importantes para el desarrollo económico futuro (Brown, DeVillez, \& Luczak, 2013).

Para la realización de la actividad, se utilizarán ejercicios impresos con imágenes de objetos 3D y de vistas normalizadas. Las figuras, para que se pueden construir en Blokify, deben estar formadas por cubos $\mathrm{y}$ no contener rampas ni elementos circulares. La tarea que se les propondrá a los alumnos es que realicen el mayor número de piezas 


\section{E K S}
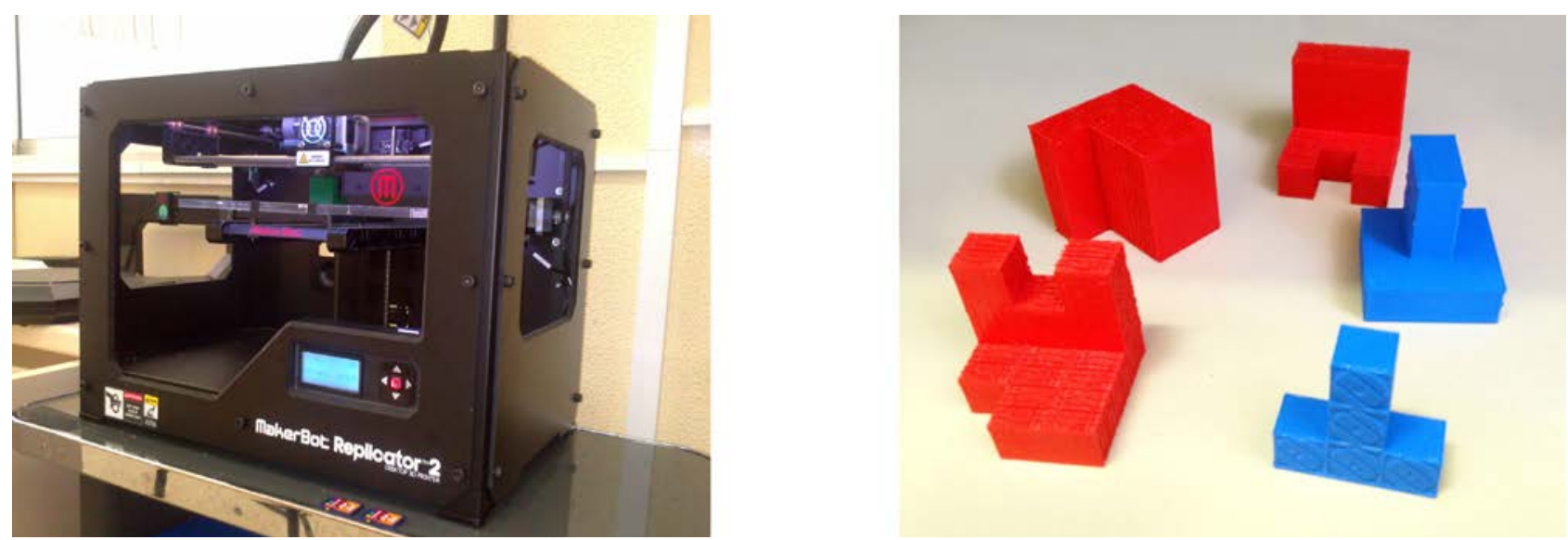

Figura 4: Figuras de Blokify impresas con una impresora 3D.

posibles en el tiempo que resta hasta finalizar

la sesión. Realizando primero una figura de los ejercicios a partir de la perspectiva $y$ después a partir de las vistas normalizadas (Figura 3).

Todas las creaciones que se realicen en Blokify se podrán imprimir con la impresora 3D (Figura 4), posibilitando así a los alumnos la opción de tocar sus creaciones. Usando las diferentes maneras de ver o percibir el objeto contribuye a la mejora de la visión espacial de los niños (Holloway, 1982).
Por lo tanto, con esta actividad los alumnos juegan con las mismas piezas pero en 4 formatos diferentes (Figura 5).

1. Dibujada sobre papel, interpretando la tridimensionalidad del objeto.

2. Dibujado sobre papel las 3 vistas de un objeto.

3. Modelado en un programa 3D con construcción a base de bloques y posibilidad de rotar el objeto en el espacio.

4. Impresión 3D del objeto diseñado. Teniendo la posibilidad de tocar un objeto físico.
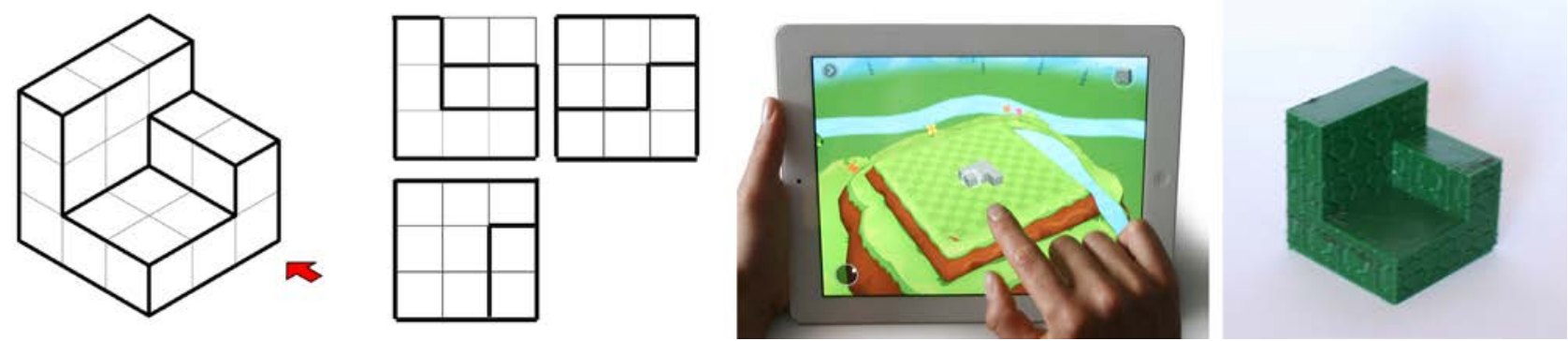

Figura 5: Diferentes formatos del mismo ejercicio

\subsection{Pottery}

Como se ha comentado, la aplicación Pottery imita el proceso de creación de figuras de arcilla en un torno. El modelado en un torno pertenece a la profesión de alfarero o ceramista, y es considerado una de las técnicas más difíciles de esta profesión. El proceso solo permite hacer figuras redondas y simétricas (Midgley, 1982), llamadas también 


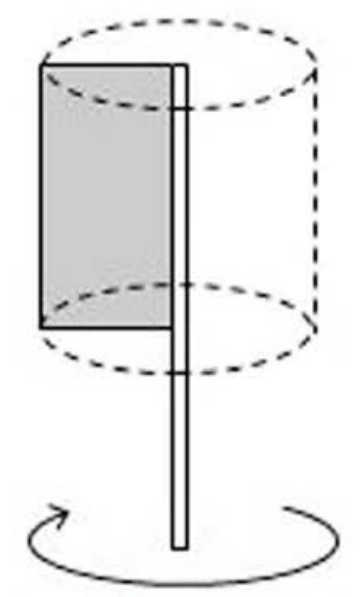

CILINDRO

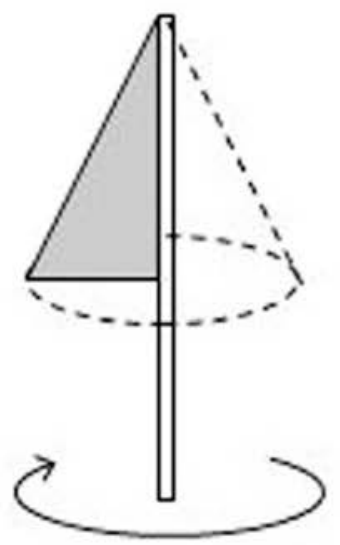

CONO

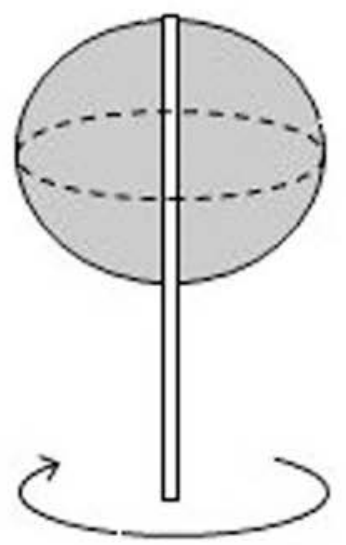

ESFERA

Figura 6: Tipo de representación de las figuras de revolución.

cuerpos o figuras de revolución. Un cuerpo de revolución es aquel que se origina al girar una figura plana alrededor de un eje y crea caras curvas. Los principales son la esfera, el cilindro y el cono. El modelado de arcilla en torno no se incluye en la programación escolar de Primaria o Secundaria, aunque sí en estudios y carreras artísticas, pero el estudio de figuras de revolución ya se encuentra incluido en el currículo desde Secundaria (BOE, 2007). La enseñanza de estas figuras habitualmente se realiza mediante dibujos del perfil y/o tridimensional de las figuras y al ser la en tres dimensiones, lo que obliga al niño a imitación de un torno siempre están rotando. imaginarse esta figura, al girar alrededor de Aunque hay que tener en cuenta que la un eje (Figura 6).

como las que se ven en la Figura 7.

El ejercicio consiste en modelar la figura con la aplicación (Figura 8), tratando que el resultado sea lo más parecido posible a la imagen dada. Atendiendo a las diferentes alturas y a la silueta dibujada. La misma actividad también es posible plantearla al revés, que los alumnos crean libremente una figura, pero que luego dibujen la silueta y el eje de rotación del cuerpo de revolución.

La aplicación cuenta con una visualización aplicación tiene ciertas limitaciones y no se

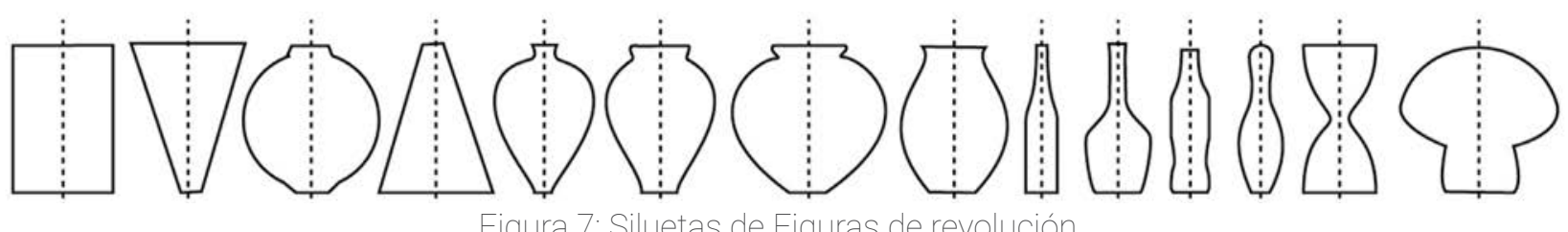

Pottery nos da la oportunidad de generar cuerpos de revolución directamente y entender a base de practicar con un juego cómo son. Como actividad a realizar con Pottery, se entregan varias siluetas de figuras dibujadas puede realizar cualquier figura.

Al principio el juego se centra más en la forma,

debido a que no tenemos todavía pinceles ni colores, por lo tanto los ejercicios partirán de una geometría. Conforme se realizan vasijas 


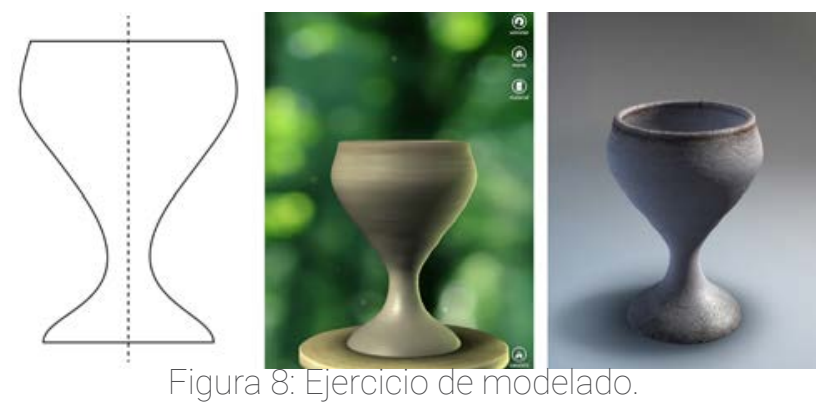

se obtienen elementos ornamentales y colores que permiten adornar las vasijas, por lo que en una segunda fase los ejercicios pueden incluir aspectos creativos que incluyan colores y diseños. Así, cada alumno podrá realizar
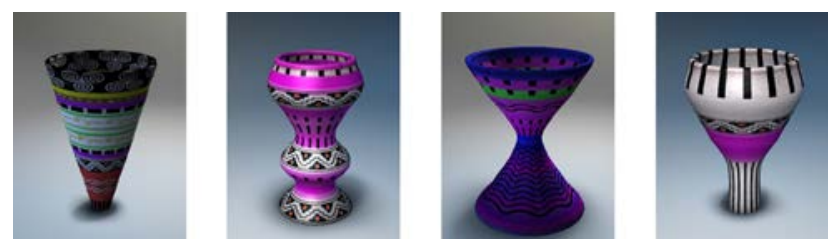

Figura 9: Posibilidades de decoración de las vasijas. una galería de imágenes con sus diseños (Figura 9), presentando estas, como parte del ejercicio.

$\mathrm{Al}$ avanzar en el juego, la misma aplicación plantea unos ejercicios a seguir. Facilita una fotografía de una vasija terminada, la cual hay que modelar y decorar exactamente igual. Dependiendo del resultado se obtiene diferentes puntuaciones (Figura 10).
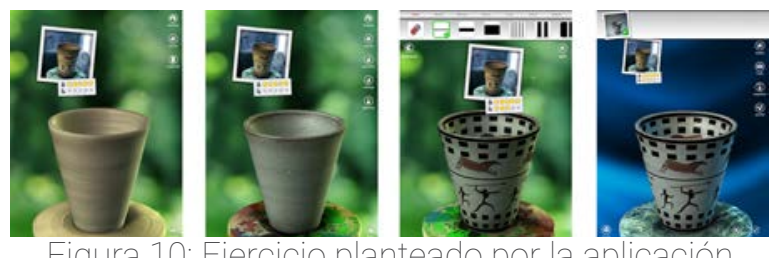

Figura 10: Ejercicio planteado por la aplicación.

\section{Conclusiones y futuros trabajos}

Blokify y Pottery, debido a que son aplicaciones en tabletas digitales, permiten introducir el modelado 3D digital en cualquier aula. Son aplicaciones aptas para iniciar a los niños en el modelado tridimensional, acostumbrándolos a trabajar y desenvolverse en un entorno 3D con medios digitales, sin requerir un aprendizaje ni conocimientos previos de programas de modelado.

Blockify puede ayudar a los alumnos, desde muy jóvenes, a entender las vistas normalizadas, el alzado, la planta y el perfil de un objeto geométrico. Este tema es importante para el dibujo técnico y su conocimiento es necesario para carreras como ingeniería o arquitectura.
Pottery se puede usar para introducir a los alumnos desde Primaria en las figuras de revolución. Nos da la posibilidad de modelar con las manos el perfil de objetos de revolución y así introducir al alumno de forma lúdica en este temario. La posibilidad de trabajar con formas orgánicas y de decorar las vasijas permite desarrollar aspectos creativos de asignaturas de dibujo, diseño y artes plásticas. Por lo tanto, como futuras actuaciones se realizarán estos talleres en entornos escolares y en diferentes niveles educativos, para de esta manera comprobar si, efectivamente, se pueden utilizar como recursos docentes en la enseñanza reglada. 


\section{Referencias}

Ball, H. G. (1978). Telegames Teach More Than You Think. Audiovisual Instruction, 23(5), 24-26.

BOE. (2007). La Ley Orgánica 2/2006, de 3 de mayo, de Educación. REAL DECRETO 1631/2006. España.

Brown, J., DeVillez, A., \& Luczak, T. (2013). Stem education coalition. Obtenido 17 de mayo, 2013, de http://www.stemedcoalition. org/

Canessa, E., Fonda, C., \& Zennaro, M. (2013). Low-cost 3D Printing for Science, Education 83 Sustainable Development. Trieste, Italy: ICTP.

Cánovas, G., García de Pablo, A., Oliaga San Atilano, A., Aboy Ferrer, I., \& Protegeles., E. (2014). Menores de Edad y Conectividad Móvil en España: Tablets y Smartphones. España: Centro de Seguridad en Internet para los Menores en España: PROTEGELES. http://www.diainternetsegura.es/descargas/ estudio_movil_smartphones_tablets_v2c. pdf.

Caño, A., Cruz, M. P., \& Solano, L. (2007). Diseño, ingeniería, fabricación y ejecución asistidos por ordenador en la construcción: evolución y desafíos a futuro. Informes de la Construcción, 59(505). http://dx.doi. org/10.3989/ic.2007.v59.i505.500

de la Torre Cantero, J., Saorín, J. L., Carbonell, C., del Castillo Cossío, M., \& Contero, M. (2012). Modelado 3D como herramienta educacional para el desarrollo de competencias de los nuevos grados de Bellas Artes. Arte, Individuo y Sociedad, 24(2), 179-193. doi: http://dx.doi.org/10.5209/ rev_ARIS.2012.v24.n2.39025

Diezmann, C. M., \& Lowrie, T. (2009). Primary students' spatial visualization and spatial orientation : an evidence base for instruction. Paper presented at the 33rd Conference of the International Group for the Psychology of Mathematics Education, Aristotle University of Thessaloniki, Greece.

Escuelapedia. (2014). Origen y evolución del tablet. Obtenido el 24 de mayo, 2014, de http://www.escuelapedia.com/origen-yevolucion-del-tablet

Gros, B. (1998). Jugando con los videojuegos: educación y entretenimiento. Bilbao: Editorial Desclée De Brouwer.

Gros, B. (2004). Pantallas, juegos y educación. La alfabetización digital en la escuela. Desclée De Brouwer.

Heinrich, P. (2012). The iPad as a tool for 
education. Kent: Naace.

Holloway, G. (1982). La concepción del espacio en el niño según Piaget. Barcelona: Paidós.

Horizon (2013). Horizon Report Edición sobre Educación Superior 2013. New Media Consortium. Austin: New Media Consortium.

Silvern, S. B. (1985-86). Classroom Use Instituto de Tecnologías Educativas. (2011). of Video Games. Educational Research Imagine: Juegos Digitales para el Aprendizaje. Quarterly, 10(1), 10-16.

http://imaginegames.eu/eng

Sjölinder, M. (1998). Spatial cognition and Long, S. M., \& Long, W. H. (1984). environmental descriptions. In N. Dahlbäck Rethinking Video Games: A New Challenge. (Ed.), Exploring Navigation: Towards a Futurist, 18(6), 35-37.

Lowery, B., \& Knirk, F. (1982). Micro- Sweden: SICS.

computer video games and spatial visualization adquisition. J. Educational Wai, J., Lubinski, D., \& Benbo, C. P. (2009). Technology Systems, 11(2), 155-166. http:// dx.doi.org/10.2190/3PAN-CHJM-RT0LW6AC

Midgley, B. (1982). Guía completa de http://dx.doi.org/10.1037/a0016127 